\title{
Editorial
}

S. O'Callaghan-Enright, B.T. Finucane

\section{Alteration of baricity: is it necessary?}

Baricity is but one of many factors which affect local anaesthetic distribution in the CSF. Greene' meticulously enumerated 25 different factors which can influence the distribution of local anaesthetics in the subarachnoid space. Of these, four factors are intimately related baricity, volume, mass, and concentration of injectate. A problem arises when one attempts to identify their individual roles, since a change in one is associated with a change in at least one other factor.

One must question the importance of baricity in relation to spinal anaesthesia. Despite the work of our predecessors, we are no closer to a scientific method of accurately determining the spread of a given injectate in the subarachnoid space. The pioneering days of spinal anaesthesia encouraged courageous experimentation but, with the introduction of thiopentone and the neuromuscular blocking drugs, general anaesthesia became a much safer undertaking. The technique of spinal anaesthesia came under attack almost simultaneously in both North America and Europe in the post-war era. The technique almost vanished from the armamentarium of the anaesthetist in the United Kingdom for more than three decades and clinical experimentation with various local anaesthetic mixtures also declined accordingly.

Most of us were introduced to the technique of spinal anaesthesia by senior colleagues who used hyperbaric solutions. We were occasional witnesses to the magic of hypobaric spinal anaesthesia. We also quickly learned that spinal anaesthesia, in contrast to general, had an additional element - unpredictability. We have all witnessed the unheralded, inexplicable failed spinal. This additional dimension forced many of us to adhere rigidly to the "tried and true" menus that we inherited.

What then are the advantages of hyperbaric spinal anaesthesia? Hyperbaric solutions are ideal when a discreet area of anaesthesia is required in a dependent area of the body. The best example of this is the classic saddle block. However, there are many practical and theoretical disadvantages to the use of hyperbaric solutions. For example, Green ${ }^{2}$ recommends a slight Trendelenberg position for optimal management of patients undergoing spinal anaesthesia. This position guarantees an increase in

University of Alberta Hospitals, Edmonton, Alberta. 
venous return but also results in a more extensive sympathetic blockade than necessary when hyperbaric solutions are used. Chamberlain ${ }^{3}$ suggests that the zone of differential blockade between sensory and sympathetic systems may be as much as 6-7 dermatones. The use of hyperbaric solutions will almost guarantee complete sympathetic interruption in the majority of cases. This physiological effect may be responsible for the rare cardiac arrest reported during spinal anaesthesia. Hyperbaric spinal anaesthesia may also be responsible for the occasional failed spinal, reported by novices, who fail to position patients quickly enough, following the injection of hyperbaric solutions. Some experts claim that they can produce strict unilateral blockade by positioning patients on one or the other side for five to ten minutes. They claim that this practice reduces haemodynamic changes to a minimum by inducing a unilateral sympathetic blockade. This claim is open to question, simply on the grounds that fixation of spinal anaesthetic solutions may take up to 45 min especially with the longer acting local anaesthetic drugs. Finally, there has been a renewed interest in continuous spinal anaesthesia due to technological advances in catheter design. Recent isolated cases of Cauda Equina Syndrome, ${ }^{4}$ associated with continuous spinal anaesthesia, has placed the technique in jeopardy once more and hyperbaric solutions are listed amongst the contributing causes of this dreadful malady.

What do hypobaric solutions offer clinicians performing spinal anaesthesia? In truth, few bother with this technique. In this issue of the journal, Bodily ${ }^{5}$ highlights the classic application of hypobaric spinal anaesthesia, i.e., performance of low rectal and anal procedures in the prone jackknife position. Lumbar punctures may be performed in this position, thereby precluding the need to move patients following the injection of local anaesthetic drugs. Apart from the novelty of performing spinal anaesthesia in this position, operating room personnel are spared the inconvenience of moving a partially paralysed patient. But, more important, this approach results in a greater degree of haemodynamic stability, simply because the Trendelenberg position is essential. However, as Bodily points out, patients must be nursed in the Trendelenberg position or at least in the supine position for at least one hour during the recovery period, and much longer, if longer-acting local anaesthetics are used.

Finally, even the most skilful technician encounters difficulty performing lumbar punctures in the prone jackknife position.

In contrast to hyperbaric spinal anaesthesia, an equal mass of an isobaric solution results in a slower onset, a less extensive blockade, a more intense motor blockade, a longer duration of action, fewer cardiovascular side effects and a lower incidence of tourniquet pain. ${ }^{6-9}$ Furthermore, the distribution of isobaric local anaesthetics are least influenced by position changes. Finally, the risk of medication error is greatly reduced because a single solution is used. The most benign error was one reported by Moore, ${ }^{10}$ who omitted to add the local anaesthetic, while performing a saddle block. We are also aware of a near tragedy involving a hypobaric spinal in which potassium chloride was used as a diluent instead of sterile water. Clearly every effort must be made to prevent such accidents. The use of a single medication goes a long way towards preventing these errors.

In summary, one suspects that our predecessors were a little misguided in their experiments with baricity. It has taken almost 100 years to realize that isobaric solutions have most of the advantages of either hypo or hyperbaric solutions, but more important, appear to be safer. Although we do not have access to dedicated isobaric solutions in Canada, many of us have found that 0.5 and $0.75 \%$ solutions of bupivacaine for epidural use, function very much like isobaric solutions, when injected into the subarachnoid space. Hopefully, the pharmaceutical industry will respond and develop a dedicated isobaric solution for spinal anaesthesia.

\section{Et la baricité, est-ce qu'on la change?}

En moins de dix années après la découverte de l'anesthésie rachidienne par Bier en 1899, Barker en introduit 1907 un nouveau concept, la baricité. En ajoutant du glucose à la cocaïne, il en modifie les propriétés et rend la solution hyperbare, ce qui lui permet de contrôler le niveau de l'anesthésie en changeant la position du patient. Cinq ans plus tard, Babcock publie son expérience des solutions hypobares sur un mélange de stovaïne et d'alcool. Pitkin fabrique une préparation hypobare, la spinocaine, mixture de procaïne et de deux neurotoxiques connus, l'alcool et la strychnine. En 1930 , Howard-Jones se met de la partie et une autre solution appelée cinchocaïne voit le jour. A la clinique Lahey, Sise continue le travail de Barber et propose la procaine hyperbare en 1928 et de tétracaïne hyperbare en 1935 . En 1933, Etherington-Wilson jette un peu plus de lumière sur la notion baricité en expérimentant sur un modèle en verre du canal rachidien; il peut ainsi étudier les effets 
de la variation de la baricité sur des solutions d'anesthésiques locaux.

Des premières observations de Barker jusqu'à nos jours, la littérature abonde de travaux sur le sujet. Après 85 ans d'expérience en anesthésie rachidienne, il est grand temps de faire le point sur l'importance de la baricité en clinique. Qu'est-ce que veut dire le terme baricité? Par définition, c'est le rapport entre la densité d'une substance donnée et la densité du liquide céphalorachidien. Ce rapport permet de déterminer la distribution des solutions anesthésiques dans le liquide céphalorachidien (LCR). Le LCR a une densité de 1,0003 et une baricité de 1,0000 à $37^{\circ} \mathrm{C}$. Une solution isobare a donc une baricité de 1,000, une solution hypobare, une baricité de moins de 1,000 et une solution hyperbare une baricité de plus de 1,000 . Pour que cette équation soit valide, il est essentiel que la densité de chacun des éléments soit mesurée à $37^{\circ} \mathrm{C}$ et que la mesure soit exprimé à la quatrième décimale, détail souvent ignoré des chercheurs cliniciens. Très peu d'études cliniques satisfont à ces critères rigides, ce qui en rend l'interprétation difficile, si ce n'est impossible. Finalement, il faut réaliser que les changements de température d'un des éléments ne peuvent être extrapolée à un autre. La densité de l'eau mesurée à la température standard baisse de 1,0000 à 0,9934 lorsqu'on élève sa température à $37^{\circ} \mathrm{C}$; ceci ne signifie pas qu'un liquide différent se comportera de la même façon. La baricité n'est qu'un des facteurs qui affectent la distribution d'un anesthésique local dans le liquide céphalorachidien, Green a colligé méticuleusement 25 facteurs qui peuvent influencer cette distribution. De ceux-ci, quatre sont intimement reliés : la baricité, le volume, la masse, et la concentration de la substance injectée. Il est difficile d'identifier leur rôle respectif, parce qu'un changement intéressant un facteur en particulier s'associe toujours à un changement dans au moins un autre facteur.

Nous devons, malgré tout, nous interroger sur l'importance de la baricité en rachianesthésie. Malgré les efforts de nos prédécesseurs, personne n'est encore arrivé à déterminer scientifiquement l'aire de distribution d'une solution injectée dans l'espace sous-arachnoïdien. A l'ère des pionniers, l'anesthésie rachidienne faisait l'objet d'une recherche intensive mais avec l'arrivée du thiopental et des myorelaxants, l'anesthésie générale est devenue une entreprise beaucoup plus fiable. A la période de l'après-guerre, la rachianesthésie a essuyé de vigoureux assauts tant en Amérique du nord qu'en Europe. La technique est pratiquement disparue de la sélection anesthésique au Royaume-Uni pour plus de trois décennies et l'expérimentation avec les mixtures anesthésiques locales a décru en conséquence.

Pour la plupart, nous avons été initiés à la rachianes- thésie par nos aînés qui utilisaient des solutions hyperbares. Nous avons aussi à l'occasion été émerveillés par les bienfaits de la rachianesthésie hypobare. Nous avons appris bien assez tôt, par ailleurs, que la rachianesthésie, contrairement à l'anesthésie générale pouvait être complètement imprévisible. Nous avons tous été témoins d'une rachianesthésie ratée, autant inattendue qu'inexpliquée. Cette dimension défavorable en a forcé plusieurs à adhérer aux recettes éprouvées et sûres qui font partie de l'héritage de l'anesthésiste moderne.

La rachianesthésie hyperbare présente-t-elle des bénéfices? Les solutions hyperbares sont idéales quand un territoire d'anesthésie limité est requis dans une région déclive. Le meilleur exemple en est l'anesthésie en selle. Il existe cependant plusieurs désavantages théoriques et pratiques à l'utilisation des solutions hyperbares. Par exemple, Green ${ }^{2}$ recommande un léger Trendelembourg pour procurer des soins optimaux aux patients anesthésiés de cette façon. Cette position favorise le retour veineux mais entraine un blocage sympathique plus étendu que nécessaire. Chamberlain suggère que la niveau de blocage sympathique peut excéder de 6-7 dermatomes la zone sensorielle. L'usage des solutions hyperbares conduit donc presque inévitablement à un bloc sympathique complet dans la majorité des cas. Cet effet physiologique peut être responsable de l'arrêt cardiaque occasionnellement rapporté après la mise en marche de la rachianesthésie. L'hyperbaricité peut aussi expliquer la rachianesthésie ratée occasionnellement le novice ne réussissant pas à mettre son patient assez rapidement en position après l'injection de la solution. Quelques experts prétendent qu'ils peuvent produire un bloc unilatéral limité en gardant leur patient sur un coté ou l'autre pour cinq à dix minutes après l'injection. Ils allèguent que la pratique du blocage unilatéral réduit les changements hémodynamiques au minimum. Cette affirmation demeure fort discutable car la solution anesthésique rachidienne peut prendre jusqu'à 45 minutes pour se stabiliser, spécialement si on utilise des produits à longue durée d'action. Finalement, nous assistons à un intérêt renouvelé pour la rachianesthésie continue grâce aux progrès technologiques réalisés dans la fabrication des cathéters. Quelques rares cas de syndrome de la queue de cheval associés à la rachianesthésie continue, ont mis l'avenir de la technique en danger une fois de plus et on ne peut ignorer que l'hyperbaricité des solutions est sur la liste des causes de cette terrible affection.

Quel est l'intérêt de la solution hypobare pour le praticien de l'anesthésie sous-arachnoïdienne ? Si on y regarde de près, il semble qu'il soit plutôt réduit. Dans cette édition du Journal, Bodily ${ }^{5}$ souligne une application pratique de la rachianesthésie hypobare, c'est-à-dire la réalisation d'interventions baisses, rectales ou anales, en 
décubitus ventral, avec le siège en position proclive. La ponction lombaire peut se réaliser dans cette position, ce qui évite le déplacement du patient après l'injection de l'anesthésique local. En plus du spectacle inusité qu' offre la réalisation d'une ponction lombaire dans cette opposition, on évite au personnel de la salle d'opération les inconvénients causés par le retournement sur la table d'opération d'un malade à demi-paralysé. Mais ce qui est plus important, avec cette approche, c'est qu'on obtient un plus grand degré de stabilité hémodynamique simplement parce qu'on peut maintenir le patient en Trendelembourg. Cependant, comme le fait remarquer Bodily, on doit prendre soin du patient en position de Trendelembourg ou en position couchée pour au moins une heure à la période de récupération ou encore plus longtemps, si on a utilisé un anesthésique local de longue durée. Il faut aussi remarquer que plusieurs éprouveront de la difficulté à réaliser une ponction lombaire dans la position déjà décrite.

Pour finir, il nous faut aussi parler de la rachianesthésie isobare. Comparativement à une solution hyperbare, une masse égale de solution isobare produit un début plus lent, un blocage moins étendu, un bloc moteur plus intense, moins d'effets cardiovasculaires et une incidence moins élevée de douleurs provoquées par le garrot. De plus, la distribution de l'anesthésique local dans l'espace est moins influencée par les changements de position. Finalement, le risque d'erreur médicamenteuse décroît du fait qu'une seule solution est employée. On rapporte quelques unes de ces erreurs dont la plus inoffensive pourrait être celle que rapporte Moore; l'omission de la solution anesthésique pendant la réalisation d'une anesthésie en selle; par contre une catastrophe aurait pu survenir lorsqu'on a utilisé du chlorure de potassium à la place de l'eau stérile dans la préparation une solution hypobare. De toute évidence, il faut éviter ce type d'erreurs et l'utilisation d'un seul agent anesthésique est un bon pas dans cette direction.

On peut conclure que nos prédécesseurs étaient plutôt mal informés sur les effets de la baricité. Il a fallu presque cent ans pour enfin comprendre que les solutions isobares ont la plupart des avantages des solutions hypoet hyperbares, mais ce qui est plus important, elles offrent plus de sécurité. Bien que nous n'ayons pas accès à des solutions isobares reconnues comme telles au Canada, plusieurs parmi nous trouvent que la bupivacaine 0,5 et $0,75 \%$ pour usage épidural se comporte à toutes fins pratiques comme une solution isobare une fois rendue dans l'espace sous-arachnoïdien. Nous espérons un jour voir l'industrie pharmaceutique combler cette lacune en fabriquant une solution isobare spécifique destinée à la rachianesthésie.

\section{References}

1 Greene NM. Distribution of local anaesthetic solutions within the subarachnoid space. Anesth Analg 1985; 64: 715-30.

2 Greene NM. The Physiology of Spinal Anesthesia. 3rd Ed Baltimore: Williams \& Wilkins 1981.

3 Chamberlain DP, Chamberlain BD. Changes in the skin temperature of the trunk and their relationship to sympathetic blockade during spinal anesthesia. Anesth Analg 1986; 65: 139-43.

4 Rigler $M$, Drasner $K$, Krejcie $T$, et al. Cauda equina syndrome after continuous spinal anaesthesia. Anesth Analg 1991; 72: 275-81.

5 Bodily $M N$, Carpenter $R L$, Owens BO. Lidocaine 0.5\% spinal anaesthesia: a hypobaric solution for short-stay perirectal surgery. Can J Anaes 1992; 39: 000-000.

6 Phelan DM, MacEvilly $M$. A comparison of hyperbaric and isobaric solutions of bupivicaine for subarachnoid block. Anaesth Intensive Care 1984; 12: 101-7.

7 Bridenbaugh PO, Hagenouw RR, Gielen MJ, Edstrom $H H$. Addition of glucose to bupivicaine in spinal anaesthesia increases incidence of tourniquet pain. Anesth Analg 1986; 65: 1181-5.

8 Axelsson KH, Widman $G B$, Sandberg AE, Hullgren S. A double-blind study of motor blockade in the lower limbs. Studies during spinal anaesthesia with hyperbaric and glucose free $0.5 \%$ bupivicaine. $\mathrm{Br} \mathrm{J}$ Anaesth 1985; 57 : 960-70.

9 Axelsson $K$, Widman GB. A comparison of bupivicaine and tetracaine in spinal anaesthesia with special reference to motor block. Acta Anaesthesiol Scand 1985; 29: 79-86.

10 Moore DC. Factors affecting spinal anaesthesia. Regional Anaesthesia 1982; 7: 20-5. 